\title{
Low-level Grouping of Straight Line Segments
}

\author{
A. Etemadi \\ J-P. Schmidt, G. Matas, J. Illingworth, and J. Kittler \\ Department of Electronic and Electrical Engineering, University of Surrey \\ Guildford, United Kingdom
}

\begin{abstract}
In this paper we present a formalism for the formation of self consistent, hierarchical, "Low-Level" groupings of pairs of straight line segments from which all higher level groupings may be derived. Additionally, each low-level grouping is associated with a "Quality" factor, based on evidential reasoning, which reflects how much the groupings differ from mathematically perfect ones. This formalism has been incorporated into algorithms within the "LPEG" software package produced at the University of Surrey. LPEG was developed as part of the Vision As Process [Crowley et al., 1989] project. We present results of the application of these algorithms to sets of line segments extracted from a test image.
\end{abstract}

\section{Introduction}

Grouping of straight line segments has been the subject of much investigation. Most research in this field has been concentrated on forming perceptually significant groupings. The reader is therefore referred to commonly available bibliographic databases on the subject with special reference to [Weiss et al., 1986], [Lowe 1987], [Mohan et al., 1989], [Horaud et al., 1990], and [Faugeras, 1990] and references therein. Many types of groupings of two or more line segments have been proposed in the literature. In general the proposed groupings either fail to allow the consistent formation of higher level groupings or involve heuristics.

Let us first consider the possible relationship between any pair of lines. Clearly they may either be collinear, parallel, or intersecting. When we consider line segments we may further subdivide the parallelism relationship into overlapping, and non-overlapping. The intersecting pairs may also be divided according to whether the intersection point lies on either, both, or only one of the line segments. These then are the complete set of relationships between two line segments. Clearly all higher level groupings may be formed by combining subsets of this set.

In this paper it is our aim to first define a formalism for the formation of this set, and show how this formalism allows us to implement these groupings in a manner useful for vision. In the first section we discuss what properties are imposed on algorithms for the formation of these groupings if they are to be useful for vision purposes. In the following sections we discuss each of the proposed groupings in turn. Associated with each grouping is a "Quality" factor based on comparing the observed grouping with an ideal case. This factor greatly 
simplifies the control of image interpretation tasks and is defined separately for each grouping in turn. We present results obtained using the "LPEG" software package within which these algorithms have been implemented. Finally we summarize our findings, and discuss this work in the context of a complete vision system.

\section{Low-Level Groupings}

The most desirable feature of any proposed grouping is consistency. Strictly mathematical definitions of parallelism, collinearity, and intersection ensure this property. However so long as our definitions are consistent we need not adhere to these mathematical definitions. We may choose for example to label any pair of line segments forming an acute angle of 10 degrees as "parallel". To ensure consistency we define "non-parallel" segments as those forming acute angles greater than 10 degrees.

Self consistency is an essential requirement since it ensures that the proposed grouping relations are independent of the order in which the line segments are chosen. For the purposes of computer vision, scale independence is another important requirement, since in general the conversion factor between distances in pixels measured across an image is not known or is poorly defined. Finally we also wish to be able to form a hierarchy of groupings which will allow us to filter unlikely combinations at an early stage.

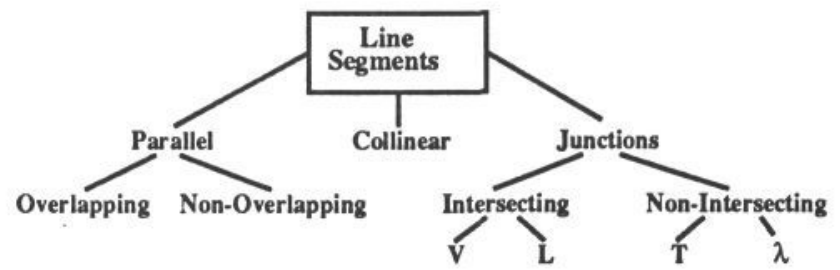

Figure 1: Low-Level groupings within the LPEG system

Based on these requirements we have defined the Low-Level groupings shown in the form of a tree structure in Figure 1. The set of straight line segments forms the top level of the tree. In the first level there are the three main groupings, namely Parallel, Collinear, and Junction. At the next level the Parallel grouping is separated into two further types based on whether the line segments overlap. The Junctions are also separated at this level into Intersecting and Non-intersecting types, based on whether the junction point resides on either, both, or neither of the line segments forming the junction. The most important distinctions are between the groupings at this level of the tree. The Junction types are finally separated to form the lowest level of the tree according to the acute angle between the line segments forming the junction. The characters used to denote the junction types also reflect their form. These junction types are especially useful when considering particular applications. For example if we know apriori that the image may contain an L shape, such as for character recognition purposes, the inclusion of $\mathrm{L}$ junctions as part of the hierarchy will allow us to rapidly identify this structure. The distinction between intersecting and non-intersecting junction types is an important one since it also allows 
us to hypothesize junctions between surfaces. Due to the generally poor segmentation the formation of all these groupings requires a statistical model of the feature extraction process, or at least a worst-case knowledge of the errors involved.

The next four sections are devoted to the discussion of the various groupings at the lowest levels of the tree. In each case we present, and discuss, the definition used in the formation of the grouping in the context of the above requirements. Associated with each grouping is a "Quality" factor which enables us to filter unlikely groupings at an early stage. We also show how this "Quality" factor is computed and discuss its relevance in each case.

\subsection{Overlapping Parallel Line Pairs}

When the acute angle between any pair of straight line segments is below a specified value, the line segments are considered as candidates for parallelism or collinearity. Note that so long as we are consistent, this value is not important. We have chosen a value of 0.06 Radians for this angle based on digital straight line properties. Having filtered line segments which may form parallel or collinear pairs, using the acute angle criterion alone, we now show how these pairs may be classified as overlapping parallel.
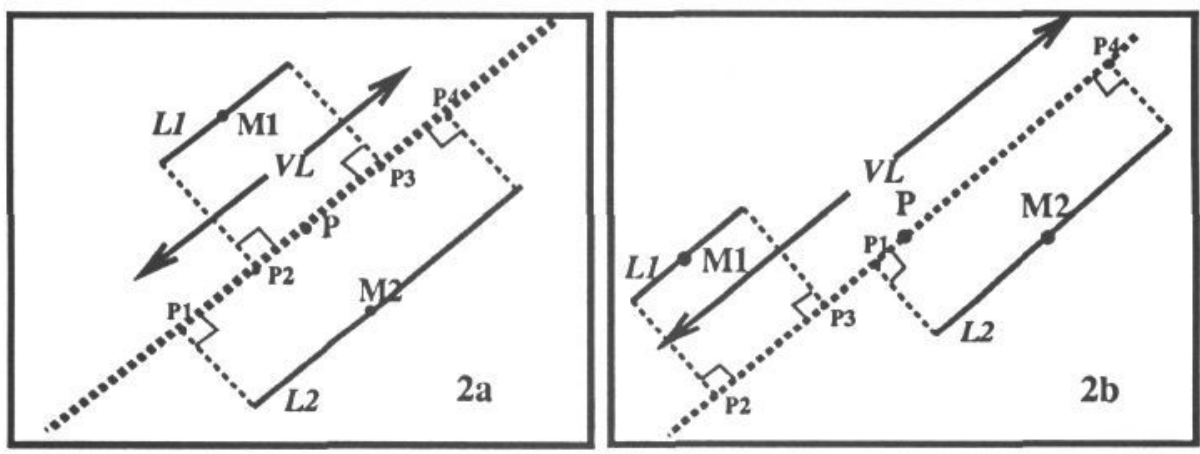

Figure 2: Overlapping (2a) and non-overlapping (2b) parallel line segments

In the following discussion we shall make use of the parameters $L_{i}, L P_{i}, \theta_{i}$, $\sigma_{i}^{\|}$, and $\sigma_{i}^{\perp}$, which represent the length, the projected length unto the "Virtual Line", orientation angle, and standard deviation of the position of the end points of the line segment along and perpendicular to its direction, respectively. The subscript $i$ is used for referencing the line segments. In general the standard deviations are used as a means of incorporating the uncertainties in the line segment extraction process into the labeling of the groupings at the lowest level of the grouping tree. These standard deviations may be replaced by constants without affecting the basic grouping algorithms. In order to avoid ambiguity in the formation of the groupings we have restricted our analysis to those line segments whose length is greater than the largest sum of any combination of their $\sigma_{i}^{\|}$, and $\sigma_{i}^{\perp}$.

Given the line segment pair $L 1$ and $L 2$ in Figure 2 a we first attempt to find the "Virtual Line" $V L . V L$ is initially defined through its orientation and the 
point $\mathbf{P}$ through which it passes. The orientation angle of the $V L$ is given by the weighted mean of the orientation of the two line segments as defined by the equation:

$$
\theta_{V L}=\frac{L_{1} \times \theta_{1}+L_{2} \times \theta_{2}}{L_{1}+L_{2}}
$$

Note that we have not used the standard deviation of the orientation angles of the line segments in the above equation since they are generally a function of the line length. Now the $\mathbf{x}, \mathrm{y}$ position of the point $\mathbf{P}$ through which the Virtual Line passes are similarly defined by:

$$
x_{V L}=\frac{L_{1} \times x_{1}+L_{2} \times x_{2}}{L_{1}+L_{2}} \text { and } y_{V L}=\frac{L_{1} \times y_{1}+L_{2} \times y_{2}}{L_{1}+L_{2}}
$$

where $x_{i}$, and $y_{i}$ indicate the $\mathrm{x}, \mathrm{y}$ positions of the midpoints, $\mathrm{Mi}$, of line $\mathrm{Li}$, respectively. We now compute the positions of the points P1, P2, P3, and P4 which as defined by the intersection points of perpendiculars dropped from the end points of the line segments onto the Virtual Line. The end points of $V L$ are defined by the pair of points $\mathrm{Pi}$, and $\mathrm{Pj}$, from the set $\mathrm{P} 1, \mathrm{P} 2, \mathrm{P} 3$, and $\mathrm{P} 4$, separated by the largest distance. These points define the length $L_{1,2}^{V L}$ of the Virtual Line. The line segments L1, and L2 are defined to be overlapping parallel if

$$
L_{1,2}^{V L} \leq L P_{1}+L P_{2}+\sigma_{1}^{\|}+\sigma_{2}^{\|}
$$

The symmetrical nature of the definition of such a grouping ensures self consistency.

If the sum of the lengths of the two line segments is equal to twice the length of the Virtual Line, and the orientations of the line segments are equal these segments form a perfect overlapping parallel pair in a strictly mathematical sense. For vision purposes we wish to determine how closely the observed pair deviates from this perfect pairing. Essentially we are attempting to determine how much evidence is available for such a perfect pairing by comparing measured quantities with the ideal case. We now define a "Quality" factor for overlapping parallel line segments which allows such a determination.

$$
\text { Quality }_{O V}=\frac{L P_{1}+L P_{2}-\sigma_{1}^{\|}-\sigma_{2}^{\|}}{2.0 \times L_{1,2}^{V L}}
$$

Our definition also ensures that this dimensionless parameter is always in the range zero to one. A value of 1.0 for the Quality factor implies a perfect grouping. This form of the definition implies that the Quality factor for parallel overlapping line segments will generally be $\geq 0.5$. The above definition is again self consistent and has the desirable property that it degrades monotonically as we move further away from the ideal case. Finally, as we shall shortly see, the computation of the Quality factor for groupings involving more than two line segments becomes greatly simplified.

The Quality factor, independent of the associated grouping, allows us to condense a great deal of information. Since this factor has the specific meaning above, it may be used for quickly indexing other types of symmetries or asymmetries within the grouped set by simply calculating the required range in Quality factor.

Sets of N overlapping parallel line segments in which all possible pairings satisfy the overlapping parallelism criterion may be combined to form a larger 
set which we shall call an overlapping parallel bundle of order $\mathrm{N}$. The associated Quality factor is defined by:

$$
\text { QualityoVP }=\frac{\sum_{j}^{N} \sum_{i>j}^{N} L P_{i}+L P_{i}-\sigma_{i}^{\|}-\sigma_{j}^{\|}}{2.0 \times \sum_{j}^{N} \sum_{i>j}^{N} L_{i, j}^{V L}}
$$

The denominator is essentially twice the sum of the lengths of the Virtual Lines associated with each pair. This form of the definition for bundles retains the self consistency property associated with pairs as described above. Note that for the Quality factor to be meaningful for arbitrarily large bundles, the choice of the type of overlapping parallel pairs must be uniform ie, all fully overlapping, or all partially overlapping. If we store the Virtual Line parameters for the overlapping parallel pairs we may compute this new quality factor directly from information already available.

\subsection{Non-overlapping Parallel and Collinear Pairs}

Given the frame work described in the last section, the definition of nonoverlapping parallel lines follows naturally. In addition to the criterion involving the acute angle between the two line segments, non-overlapping parallel lines must also satisfy

$$
L_{1,2}^{V L}>L P_{1}+L P_{2}+\sigma_{1}^{\|}+\sigma_{2}^{\|}
$$

and the perpendicular distances from the point $\mathbf{P}$ in Figure $2 \mathrm{~b}$ to the lines of which $L 1$ and $L 2$ are segments must be greater than the largest standard deviation in the position of the line segments perpendicular to their direction $\left(\sigma_{i}^{\perp}\right)$. This additional criterion allows us to distinguish between non-overlapping parallel, and collinear line segment pairs. A collinear pair is essentially a modified non-overlapping parallel pair such that the perpendicular distance from the point $\mathbf{P}$ to both line segments is less than or equal to $\sigma_{i}^{\perp}$.

We define a perfect non-overlapping parallel pair as one for which the sum of the lengths of the line segments is equal to the length of the Virtual Line, and the orientations of the line segments are equal. The Quality factor for this grouping is given by

$$
\text { Quality }_{N O V P}=\frac{L P_{1}+L P_{2}-\sigma_{1}^{\|}-\sigma_{2}^{\|}}{L_{1,2}^{V L}}
$$

We may also form bundles of $\mathrm{N}$ non-overlapping parallel line segments, using the same pairwise criterion as for overlapping parallel lines. In this case however equation 5 is no longer appropriate since the Quality factor for the bundle would inevitably decrease as we increase the number of lines forming the bundle. The appropriate form which still retains the self consistency, and monotonicity property associated with the Quality factor for parallel overlapping bundles is:

$$
\text { Quality }_{N O V P}^{N}=\frac{\sum_{j}^{N} \sum_{i>j}^{N} L P_{i}+L P_{i}-\sigma_{i}^{\|}-\sigma_{j}^{\|}}{\max _{i \neq j} L_{i, j}^{V L}}
$$

The choice of the type of bundle (step like, or staggered) is application specific. Bundles of collinear lines and their associated Quality factor are also formed in the same way. Pairs and bundles of collinear lines may be replaced by their associated Virtual Line and such lines may be treated as physical lines for the purpose of performing further grouping operations. 


\section{$2.3 \quad \mathrm{~V}$ and $\mathrm{L}$ Junctions}

A V junction is defined as any pair of line segments which intersect, and whose intersection point either lies on one of the line segments and is less than $\sigma_{i}^{\|}+\sigma_{j}^{\perp}$ away from the end points of the line segment, or does not lie on either of the line segments. An additional requirement is that the acute angle between the two lines must lie in the range $\theta_{\min }$ to $\theta_{\max }$. In order to avoid ambiguity with parallel or collinear pairs, $\theta_{\min }$ is chosen to be the same as the limiting angle used to filter line pairs forming possible parallel or collinear groups. L junctions are a special case of a $\mathrm{V}$ junction where $\theta_{\min }$ for $\mathrm{L}$ junctions is greater than $\theta_{\max }$ for the $\mathrm{V}$ junctions, and $\theta_{\max }$ for $\mathrm{L}$ junctions is $\frac{\pi}{2}$. In order to avoid ambiguity with $\lambda$ junctions we also label as $\mathrm{V}$ junctions any line segment pairs, satisfying the above criteria for $\mathrm{V}$ junctions, the distance between whose closest end points is less than the larger of $\sigma_{i}^{\|}$or $\sigma_{i}^{\perp}$.

Now a perfect $\mathrm{V}$ junction is defined as one in which the intersection point $\mathbf{P}$, shown in Figure 3a, lies precisely at the end points of the line segments. Note that there are now two Virtual Lines which share the end point $\mathbf{P}$. The points $\mathrm{P} 1$, and P4, denote the remaining end points of the Virtual Lines respectively. We now define the Quality factor as

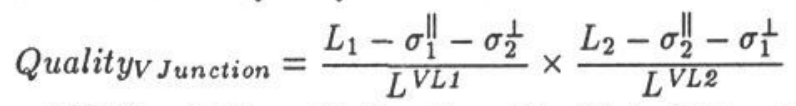

where $L^{V L i}(i=1,2)$ are the lengths of the Virtual Lines VL1 and VL2 displayed in Figure 3a. In this case we have chosen to multiply the ratios of the lengths of the lines to the lengths of the Virtual Lines since we are trying to penalize pairings in which either line is far away from the junction point. The Quality factor nevertheless retains the symmetry property described for parallel line segment pairs. The Quality factor for L junctions is defined in precisely the same manor.
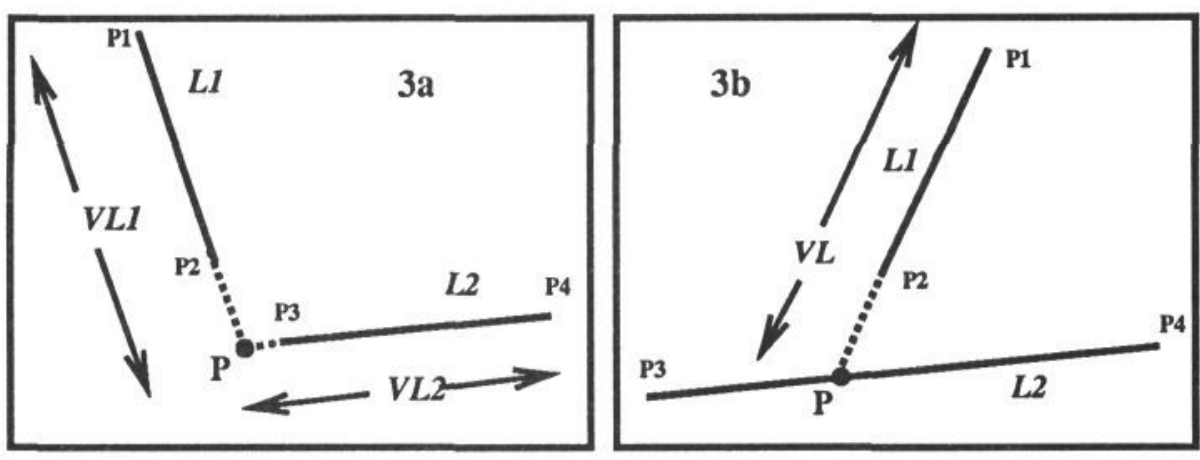

Figure 3: V (3a) and $\lambda(3 \mathrm{~b})$ Junctions

Since an infinity of shapes may be created using $\mathrm{V}$ junctions, the concept of Quality as applied to an arbitrary bundle of $\mathrm{N}$ junctions becomes meaningless. For closed sets of junctions however, such a definition is possible. The formation of the closed sets is much simplified by flagging the end points of the lines as the closer to, and the further away from, the junction point, respectively. The flagging of the end points simplifies the the search for other types of bundles 
such as triplets of lines sharing a common junction point. The Quality factor for closed sets of combinations of $\mathrm{V}$ and $\mathrm{L}$ junctions, denoted as $S_{N}$ where $\mathrm{N}$ is the number of lines in the set, is defined so as to reflect the amount of missing information in the hypothesized closed set. The precise steps in the formation of the closed sets is outside the scope of this article. However it suffices to say that all relevant junction points required to close the set (eg. to form a square or hexagon etc..) are first computed. This then allows us to compute the circumference of the hypothesized perfect closed set, and hence the Quality factor as

$$
\text { Quality }_{S_{N}}=\frac{\sum_{j}^{N} \sum_{i>j}^{N} L_{i}-\sigma_{i}^{\|}-\sigma_{j}^{\perp}}{2.0 \times \text { Circumference }}
$$

Where the subscripts $i, j$ refer only to those pairs of line segments which form the sides of the closed set. Note that collinear line segment pairs may be also be used in the formation of the closed set.

\section{$2.4 \lambda$ and T Junctions}

A $\lambda$ junction is one where the intercept point lies on one of the line segments, and the line segments do not form a $\mathrm{V}$ or L Junction. Choosing $\theta_{\min }$ and $\theta_{\max }$ so that they have the same values as those used for $\mathrm{V}$ Junctions assures consistency. $\mathrm{T}$ junctions are a special case of $\lambda$ junctions where $\theta_{\min }$ for $\mathrm{T}$ junctions is equivalent to $\theta_{\max }$ for the $\lambda$ junctions, and $\theta_{\max }$ for $\mathrm{T}$ junctions is $\frac{\pi}{2}$. There is only one Virtual Line involved in the definition of $\lambda$ and T junctions as shown in Figure 3b. A perfect $\lambda$ or T junction is one in which the intersection point lies precisely at the end point of only one of the line segments. We define the Quality factor for $\lambda$ junctions as

$$
\text { Quality }_{\lambda \text { Junction }}=\frac{L_{1}-\sigma_{1}^{\|}-\sigma_{2}^{\frac{1}{2}}}{L_{V L 1}}
$$

where $L_{1}$ is the length of the line which does not include the junction point, and $L_{V L 1}$ is the length of the Virtual Line. The Quality factor for T junctions is defined in precisely the same manor as for $\lambda$ Junctions. It is more important that we do not mislabel possible $\mathrm{V}$ or $\mathrm{L}$ junctions as $\lambda$ or $\mathrm{T}$ junctions, than vice versa, since the latter may be easily rectified when we form higher level groupings, but the former tends to propagate to higher levels. This is the main reason for the inclusion of the additional criterion, based on proximity of end points, stated in the last section.

\section{Experimental Results}

The above groupings have been implemented within the LPEG system. The form of the definitions of the groupings allows many of the computations to be performed in parallel. In addition each of the groupings themselves may be computed independently. The best implementation of LPEG would therefore be in a massively parallel environment. The left-most image in Figure 4, kindly supplied by Dr. R. Horaud at the LIFIA institute in Grenoble, is that of a Widget used for test purposes. The next two images are the results of the Canny edge detector, and annotated extracted line segments using the Hough transform, respectively. The extracted line segments have been allocated an 
identification number which is displayed as the annotation in the corresponding image.
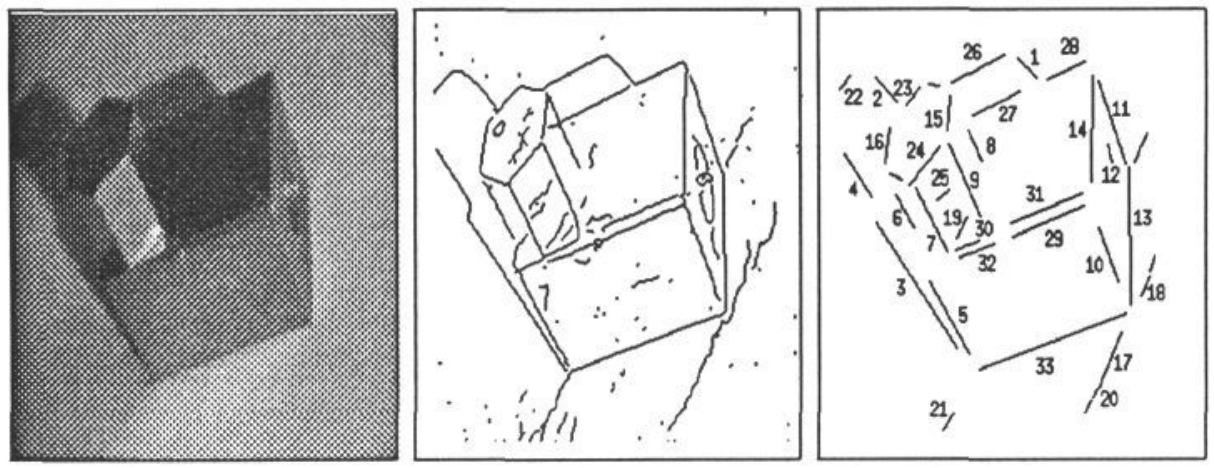

Figure 4: Image used for testing "Low-Level" grouping algorithms

The results of groupings with associated Quality factor greater than or equal to 0.5 , and using a limiting angle of 0.06 radians, are displayed in Table 1. The length of the line segments extracted using the Hough transform were constrained to be above 10 pixels. Starting with an ASCII list of 39 extracted line segments the grouping was performed in $0.66 \mathrm{CPU}$ seconds (excluding I/O) on a Sun SLC. Each column in the table contains the identification numbers associated with line segments forming the grouping type indicated at the top of the table. In order to show how the Quality factor may be used effectively in filtering unlikely groupings, we have applied the algorithm to the same set of lines and limiting angles used in the formation of Table 1, but using a limiting Quality factor of 0.3 . The results are presented in Table 2 and the corresponding processing time was $0.57 \mathrm{CPU}$ seconds.

\section{Summary and Conclusions}

We have also applied the algorithm to other lists of line segments extracted from images of various indoor scenes. The computation time, using the same limiting angle and, for limiting Quality factors of 0.5 , range from 3 to 60 seconds for a lists containing approximately 100 to 320 line segments. In conclusion we believe that the formalism presented is sufficiently flexible that it may be used in varied applications in which the user wishes to extract a limited set of groupings from a set of line segments. The most important attribute of the proposed groupings is the Quality factor. This parameter allows us to handle the combinatorial explosion of groupings by focusing the attention of the system on those groupings which are near ideal. In general we would initially look for high Quality groupings, and use these to initiate new image understanding tasks. Since we may determine a worst-case time scale for the formation of the groupings, given the number of line segments in the image, the resources which need to be allocated to the grouping process, and the limiting Quality factor, may be easily determined. Current work involves the construction of the intermediate level of the LPEG system which uses these groupings in the formation of closed sets. 


\section{References}

[Crowley et al., 1989] J.L. Crowley, A. Chehikian, J. Kittler, J. Illingworth, J.O. Eklundh, G. Granlund, J. Wiklund, E. Granum and H.I. Christensen, Vision as Process Technical Annex ESPRIT-BRA 3038, University of Aalborg, 1989 .

[Faugeras, 1990] O. Faugeras (Ed.), Computer Vision, ECCV 90.

[Horaud et al., 1990] R. Horaud, and F. Veillon, Finding geometric and relational structures in an image, First ECCV, 52, 57-77, 1990.

[Lowe 1987] D.G. Lowe, Three-dimensional object recognition from single twodimensional images, AI 31, 355-395, 1987.

[Mohan et al., 1989] R. Mohan and R. Nevatia, Using perceptual organization to extract 3-D structures, T-PAMI 11, 1121-1139, 1989.

[Weiss et al., 1986] R. Weiss and M. Boldt, Geometric grouping applied to straight lines, CVPR, 489-495, 1986.

\begin{tabular}{|c|c|c|c|c|c|c|}
\hline OVP & NOVP & Collinear & L & V & V (cont $^{d}$.) & $\lambda$ \\
\hline 67 & 34 & 2728 & 1029 & 333 & 1317 & 1329 \\
\hline 78 & 45 & 2932 & 1128 & 533 & 1331 & 1720 \\
\hline 79 & 56 & 3031 & & 624 & 1333 & \\
\hline 89 & 57 & & & 724 & 1428 & \\
\hline 1314 & 1011 & & & 730 & 1429 & \\
\hline 2224 & 1516 & & & 732 & 1431 & \\
\hline 2627 & 1819 & & & 915 & 1526 & \\
\hline 2729 & 2223 & & & 924 & 1733 & \\
\hline 2931 & 2324 & & & 931 & & \\
\hline 2933 & 2628 & & & 932 & & \\
\hline 3032 & 2829 & & & 1033 & & \\
\hline 3033 & 2831 & & & 1113 & & \\
\hline 3133 & 2930 & & & 1114 & & \\
\hline 3233 & 3132 & & & & & \\
\hline
\end{tabular}

Table 1: Results of grouping of line segment features extracted from the image presented in Figure 9. A limiting Quality factor of 0.5, was used to filter the groupings. No T junctions were found.

\begin{tabular}{|c|c|c|c|c|c|}
\hline OVP & NOVP & Collinear & L & V & $\lambda$ \\
\hline 2224 & 34 & 2932 & & 1113 & \\
\hline 2627 & 57 & & & 1333 & \\
\hline 2729 & 1516 & & & & \\
\hline 2931 & 2324 & & & & \\
\hline & 2829 & & & & \\
\hline & 2831 & & & & \\
\hline
\end{tabular}

Table 2: A limiting Quality factor of 0.8 , was used to filter the groupings. 